\title{
Cosmic ray transport in the proximity of pulsars and the formation of gamma-ray halos
}

\section{S. Recchia, ${ }^{a, b, *}$ M. Di Mauro, ${ }^{b}$ F. A. Aharonian, ${ }^{c, d}$ F. Donato, ${ }^{a, b}$ S. Gabici ${ }^{e}$ and S. Manconi $^{f}$}

${ }^{a}$ Department of Physics, University of Torino, via P. Giuria, 1, 10125 Torino, Italy

${ }^{b}$ Istituto Nazionale di Fisica Nucleare, via P. Giuria, 1, 10125 Torino, Italy

${ }^{c}$ Dublin Institute for Advanced Studies, 31 Fitzwilliam Place, Dublin 2, Ireland

${ }^{d}$ Max-Planck-Institut für Kernphysik, Postfach 103980, D-69029 Heidelberg, Germany

${ }^{e}$ Université de Paris, CNRS, Astroparticule et Cosmologie, F-75006 Paris, France

${ }^{f}$ Institute for Theoretical Particle Physics and Cosmology, RWTH Aachen University, Sommerfeldstr. 16, 52056 Aachen, Germany

E-mail: sarah.recchia@unito.it, dimauro.mattia@gmail.com

The detection of an extended multi-TeV gamma ray emission around the Geminga and Monogem pulsars by the HAWC collaboration provides a unique tool to investigate the transport properties of cosmic rays in the turbulent magnetized interstellar medium. Previous analyses of such emission in the framework of pure isotropic diffusion lead to infer a suppression of the diffusion coefficient in the pulsars region by at least two orders of magnitude compared to typical values found in models of Galactic cosmic ray propagation. In this work, we investigate the transition from the ballistic to the diffusive regime, and show that, when such transition is taken into account, a good fit to the HAWC $\gamma$-ray data around Geminga and Monogem is obtained with typical values of the interstellar diffusion coefficient.

$37^{\text {th }}$ International Cosmic Ray Conference (ICRC 2021)

July 12th - 23rd, 2021

Online - Berlin, Germany

\footnotetext{
${ }^{*}$ Presenter
} 


\section{Introduction}

The detection by the HAWC collaboration of extended, above $\sim 1 \mathrm{TeV} \gamma$-ray halos around the Geminga and Monogem pulsars, provided a unique tool to investigate the propagation of veryhigh-energy (VHE) cosmic rays (CRs) in the interstellar medium (ISM) [1]. Indeed, such halos are very likely produced by runaway VHE electrons and positrons $\left(e^{ \pm}\right)$, released by the pulsar and diffusing in the surrounding medium, that produce inverse Compton scattering (ICS) photons on the interstellar radiation field. The HAWC data have been mostly analyzed in the assumption that the $e^{ \pm}$propagate isotropically and diffusively at any time after being released from the source. This lead to the conclusion [1-7] that, in order to fit the Geminga and Monogem halos surface brightness the energy dependent $\mathrm{CR}$ diffusion coefficient, $D(E)$, within few tens pc from pulsars should be at least two orders of magnitude smaller than the typical values employed in standard Galactic CR propagation models [8]. This rather unexpected outcome has since then acquired popularity but so far no satisfactory theoretical explanation has been proposed (see e.g. [9-11]). In this work we show that such conclusion on the diffusion coefficient is a consequence of the wrong assumption that the diffusive approximation applies at any time after injection. In fact, given the diffusion coefficient $D(E)$, the scattering time and mean free path are given by $\tau_{c}(E)=3 D(E) / c^{2}$ and $\lambda_{c}(E) c$ respectively (see e.g. [8]). The CR transport after injection is characterized by three different phases: i) for $t<<\tau_{c}$ propagate ballistically; ii) for $t>\tau_{c}$ the transport is diffusive; iii) a transition between the two regimes, that we call quasi-ballistic. $\tau(E)$ and $\lambda_{c}(E)$ represent the energy-dependent time and spatial scales for isotropization of the particle direction (see e.g $[12,13]$ and references therein). If applied at time-scales below $\tau_{c}$, the diffusion theory is plagued by the superluminal propagation problem [12,13]. Instead, if one takes properly into account the transition between the ballistic and diffusive regimes, the Geminga and Monogem $\gamma$-ray halos can be explained without invoking a suppression of diffusion. It can be shown that, for pulsars as Geminga and Monogem, the CR spatial distribution within $\approx \lambda_{c}$ from the source, is dominated by particles injected within the last $\tau_{c}$ [13] that move quasi-ballistally. The magnitude of $\lambda_{c}$ depends on the particle energy and on the diffusion coefficient. The $\gamma$-ray emission, detected by HAWC at energies $5-50 \mathrm{TeV}$, is mainly produced by $e^{ \pm}$of energy between $20-200 \mathrm{TeV}$ (see [14]).

The standard diffusion coefficient at these energies is $D \gtrsim 10^{29}-10^{30} \mathrm{~cm}^{2} / \mathrm{s}$ [8], which implies $\lambda_{c} \gtrsim 3-30 \mathrm{pc}$, that is comparable with the spatial extension of $\sim 10 \mathrm{pc}$ measured for the $\gamma$-ray halo around Geminga and Monogem [1]. Also at lower energies, namely $e^{ \pm}$of energy $E \sim 100-1000$ $\mathrm{GeV}$, relevant for the Fermi-LAT data analyzed by [5], $\lambda_{c} \gtrsim 3-10 \mathrm{pc}$, so that also these data should be analyzed keeping into account the ballistic-diffusion propagation.

\section{Ballistic-diffusive propagation transition}

The standard Galactic CR diffusion coefficient is typically parametrized as $D(E) \approx D_{0} E_{\mathrm{GeV}}^{\delta} \mathrm{cm}^{2} / \mathrm{s}$, where $E_{\mathrm{GeV}}$ is the particle energy in $\mathrm{GeV}, D_{0} \sim 1-4 \times 10^{28} \mathrm{~cm}^{2} / \mathrm{s}$ and $\delta \sim 0.3-0.6$ [8]. The corresponding mean free path and scattering time are given by

$$
\begin{aligned}
& \lambda_{c}\left(E_{\mathrm{GeV}}\right) \approx 0.3 D_{0,28} E_{\mathrm{GeV}}^{\delta} \mathrm{pc} \\
& \tau_{c}\left(E_{\mathrm{GeV}}\right) \approx 1.0 D_{0,28} E_{\mathrm{GeV}}^{\delta} \mathrm{yr},
\end{aligned}
$$




\begin{tabular}{|c|c|c|c|c|c|}
\hline & $\dot{E}[\mathrm{erg} / \mathrm{s}]$ & $T[\mathrm{kyr}]$ & $l[\mathrm{kpc}]$ & $\tau_{0}[\mathrm{kyr}]$ & $n$ \\
\hline Geminga & $3.25 \times 10^{34}$ & 342 & 0.19 & 12.0 & 3 \\
\hline Monogem & $3.8 \times 10^{34}$ & 111 & 0.288 & 12.0 & 3 \\
\hline
\end{tabular}

Table 1: Characteristics of Geminga [15] and Monogem (ATNF catalog: https://www . atnf. csiro . au/ research/pulsar/psrcat/): $\dot{E}$ is the present spin-down luminosity, $T$ is the age, $l$ the distance from Earth, $\tau_{0}$ the spin-down timescale and $n$ the braking index.

where $D_{0,28}$ is the diffusion coefficient at $1 \mathrm{GeV}$ in units of $10^{28} \mathrm{~cm}^{2} / \mathrm{s}$. We assume $\delta=0.5$ as a benchmark parameter, in agreement with recent analysis of CR data (see e.g., [8]). $\lambda_{c}$ increases with $D_{0}$ and with the particle energy. Larger values of $\delta$ implies larger values of $\lambda_{c}$ at high energy. For values of $D_{0} \lesssim 10^{26} \mathrm{~cm}^{2} / \mathrm{s}$ the transition would take place very close to the source and a pure diffusive model can be safely applied. The injection of $e^{ \pm}$from a pulsar of age $T$ that turns on at $t=0$ is assumed to reflect the time dependent spin down luminosity $L(t)=\eta L_{0}\left(1+\frac{t}{\tau_{0}}\right)^{(-(n+1) /(n-1))}$, where $L_{0}$ is the initial spin-down luminosity, $n$ is the braking index (assumed to be 3 ) and $\tau_{0}$ is the typical pulsar spin down timescale. Such parameters are reported in Tab. 1

Particles injected at times $t_{0} \leq T-\tau_{c}$ are treated in the diffusive regime, with the inclusion of energy losses. The electron density, $f_{\text {diff }}$, at a distance $r$ from the source of age $T$ reads (see, e.g. [5]):

$$
f_{\text {diff }}(r, E)=\int_{0}^{T-\tau_{c}} d t_{0} \frac{Q\left(E_{0}\right) L\left(t_{0}\right)}{\pi^{3 / 2} r_{d}^{3}\left(E, E_{0}\right)} \frac{b\left(E_{0}\right)}{b(E)} e^{-\frac{r^{2}}{r_{d}^{2}\left(E, E_{0}\right)}},
$$

where $b(E)=d E / d t$ is the energy loss rate, which includes ICS losses on the interstellar radiation field (see [14] for details) and synchrotron radiation losses assuming a Galactic magnetic field of $3 \mu \mathrm{G}$. Particles emitted from the source at time $t_{0}$ with energy $E_{0}$ cool down to energy $E$ during the time $T-t_{0}$, and $r_{d}^{2}\left(E, E_{0}\right)=4 \int_{E}^{E_{0}} D\left(E^{\prime}\right) / b\left(E^{\prime}\right) d E^{\prime}$ is the propagation length squared. $Q(E)$ is the injection spectrum, here modeled as a broken power-law with index below and above the break (fixed to $500 \mathrm{GeV}$ ) of 1.4 and 2.2, respectively (see also [1,5]). This spectral shape is compatible with multiwavelenght observations of PWNe but the value of the parameters are not well constrained [16]. The injection spectrum is normalized as $\int_{0}^{T} d t \int_{0.1 \mathrm{GeV}}^{\infty} E Q(E, t) d E=\eta W_{0}$, where $W_{0}$ is the total spin down energy [5,6] and $Q(E, t)$ is given by $Q(E, t)=Q(E) \cdot L(t)$.

In the ballistic regime (particles injected at times $T-\tau_{c}<t_{0} \leq T$ ), the $e^{ \pm}$density, $f_{\text {ball }}$, is given by $[12,13]$ :

$$
f_{\text {ball }}(r, E)=\int_{T-\tau_{c}}^{T} \frac{Q(E) L(T)}{4 \pi c^{3}\left(T-t_{0}\right)^{2}} \delta\left(\left(T-t_{0}\right)-\frac{r}{c}\right) d t_{0}=\frac{Q(E) L(T)}{4 \pi c r^{2}} H\left(\tau_{c} c-r\right),
$$

where $H\left(\tau_{c} c-r\right)$ is the Heaviside function, which we substitute with with $\exp \left[-\left(r /\left(2 \lambda_{c}\right)\right)^{2}\right]$ to make the solution smooth (we check that the results are not changed appreciably by the specific shape of the smoothing function). Since typically $\tau_{0}>>\tau_{c}$ (few kys against few tens yrs) and the energy loss time at the energy relevant for our analysis is larger than $\tau_{c}$, we assume a constant luminosity equal to $L(T)$ and neglect energy losses in this regime. The total $e^{ \pm}$density is given by $f_{e}(r, E)=f_{\text {ball }}(r, E)+f_{\text {diff }}(r, E)$. 

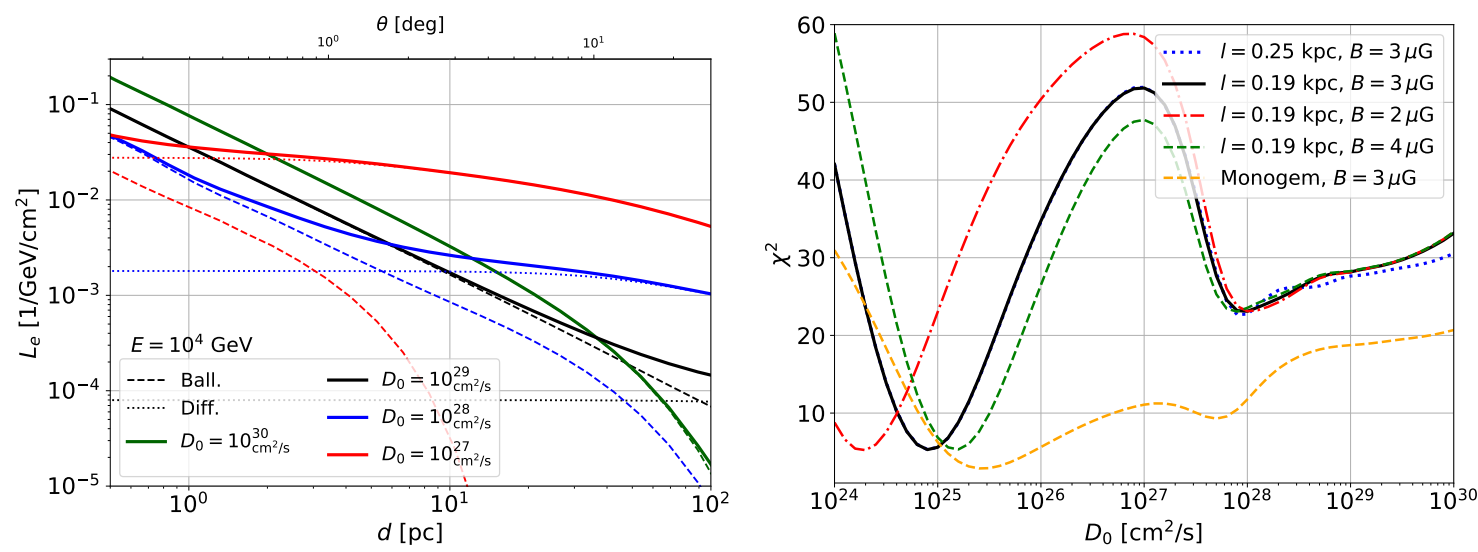

Figure 1: Left panel: electron density of the Geminga pulsar integrated along the line of sight as a function of the projected distance from the source $d$, at $10 \mathrm{TeV}$ and for different values of $D_{0}$, from $10^{27} \mathrm{~cm}^{2} / \mathrm{s}$ to $10^{30} \mathrm{~cm}^{2} / \mathrm{s}$. The dashed lines correspond to the quasi-ballistic regime contribution, the dotted line to the diffusive contribution and the continuous lines to the total. Right panel: fit to the HAWC data for Geminga and Monogem: $\chi^{2}$ as a function of the normalization $D_{0}$ of the diffusion coefficient at $1 \mathrm{GeV}$. We report the results obtained for two different distances of Geminga and for different magnetic field values $B$. For Monogem we only show the fit for our benchmark model for the source distance and magnetic field.

In the ICS process $\gamma$ rays are mainly emitted along the direction of the parent CR. In the diffusive regime the particle distribution is isotropic and the size of the $\gamma$-ray halo reflects the size of the $e^{ \pm}$halo. In the strictly ballistic regime the $\gamma$-ray source would be detected as point-like (due to particles that point toward the observer), no matter the size of the $e^{ \pm}$halo $[13,17]$. For the intermediate quasi-ballistic regime we follow the prescription proposed by [13] for the particle angular distribution $M(\mu)=\frac{1}{Z(x)} \exp \left(-\frac{3(1-\mu)}{x}\right)$, which encompasses the the small-angle diffusion approximation. Here $Z(x)=\frac{x}{3}(1-\exp (-6 / x)), x(E)=r c / D(E)=3 r / \lambda_{c}, \mu=(l \cos (\theta)-s) / r$ and $r(s, \theta)=\sqrt{l^{2}+s^{2}-2 l s \cos \theta}, s$ is the distance along the line of sight, $\theta$ the angle between the source and the line of sight, $l$ the distance from the source and $\mu$ the cosine of the angle between the radial direction and the direction of the line of sight. The complete particle distribution function reads $F_{e}(E, s, \theta)=2 f_{e}(E, r(s, \theta)) M\left(\mu(s, \theta) . M(\mu)\right.$ is normalized as $\int_{-1}^{1} M(\mu) d \mu=1$. In the limit $r>>\lambda_{c}$ the diffusive regime is recovered and $M(\mu)$ reduces to $1 / 2$. For $r<\lambda_{c}, M(\mu)$ takes into account the anisotropic angular distribution of particles in the quasi-ballistic regime.

The integral of the electron distribution function $F_{e}$ along $s, L_{e}(E, \theta)=\int_{0}^{\infty} d s F_{e}(E, s, \theta)$, as a function of the projected distance from the source $d=l \tan \theta$, reflects the spatial profile of the $\gamma$-ray emission. In Fig. 1 (left panel) we show $L_{e}$ in the case of Geminga (using the parameters of Tab. 1) and assuming a $e^{ \pm}$conversion efficiency $\eta=1$. The particle energy is fixed to $E=10 \mathrm{TeV}$ and $D_{0}$ varies in the range $10^{27}-10^{30} \mathrm{~cm}^{2} / \mathrm{s}$. Up to distances $\approx \lambda_{c}(E) / 3$ from the source, $L_{e}$ is dominated by $e^{ \pm}$injected within the last $\tau_{c}$, that move quasi-ballistically, which results in a spatial profile of $L_{e}(E, \theta)$ a bit steeper than $\propto 1 / r$, as due to the effect of $M(\mu)$ (see the discussion in [13]). At $d \gtrsim \lambda_{c}(E) / 3 L_{e}$ is dominated by particles injected at $t_{0}<T-\tau_{c}$, that move diffusively and give a rather flat $L_{e}$ profile up to a distance $d \approx r_{d} \sim \sqrt{4 D(E) t_{\text {loss }}(E)}$, where $t_{\text {loss }}$ is the time scale for energy losses. $r_{d}$ is the distance that particles can travel before loosing their energy, which reflects the exponential cut-off $\exp \left(-r^{2} / r_{d}^{2}\right)$ in the diffusive solution (see Eq. 3 and [18]). 
For $D_{0} \lesssim 10^{27} \mathrm{~cm}^{2} / \mathrm{s}$ the ballistic-diffusion transition would happen at $r<0.05 \mathrm{pc}$ and $\theta<0.1^{\circ}$ so that the $\gamma$-ray morphology observed by HAWC would be solely affected by diffusion. Instead, for $D_{0}=10^{28}-10^{29} \mathrm{~cm}^{2} / \mathrm{s}, \lambda_{c}>10 \mathrm{pc}$ and the spatial extension of the $\gamma$-ray halo is substantially determined by the quasi-ballistic propagation regime. At $D_{0} \gtrsim 10^{29} \mathrm{~cm}^{2} / \mathrm{s}$ particles of that energy move ballistically for hundreds of $\mathrm{pc}$, which would imply a more point-like source, as indicated by the appearance of a cutoff at distances $d>20$ pc. As shown by Eq. 1, a decrease(increase) of the particle energy at fixed $D_{0}$ and $\delta$ moves the transition closer(away) from the source. Moreover, a decrease(increase) of $\delta$ at fixed $D_{0}$ and $E$ moves the transition closer(away) from the source. The effect is obviously more and more prominent at larger energies.

\section{Fit to the HAWC data for Geminga and Monogem}

We apply the ballistic-diffusive model described above to the fit the HAWC data for the surface brightness of Geminga and Monogem, keeping $D_{0}$ and the $e^{ \pm}$conversion efficiency as free parameters. Our benchmark parameters are reported in Tab. 1. We also test our model for a distance of $0.25 \mathrm{kpc}$ of Geminga [19], and for different values of the Galactic magnetic field $B$ around $3 \mu \mathrm{G}$. The resulting $\chi^{2}$ as a function of such parameters is shown in Fig. 1, while in Fig. 2 we show the spatial distribution of the $\gamma$-ray flux for our best fit model to the HAWC data in the case of a purely diffusive model (suppressed diffusion) and of our ballistic-diffusion model (typical Galactic diffusion coefficient). In the case of Geminga the $\chi^{2}$ exhibits first minimum at $D_{0} \approx 0.2-2 \times 10^{25}$ $\mathrm{cm}^{2} / \mathrm{s}$ with a best-fit $\chi^{2}$ of about 5 , and a second minimum at $D_{0} \approx 0.7-2 \times 10^{28} \mathrm{~cm}^{2} / \mathrm{s}$ with a best-fit $\chi^{2}$ of about 22 . The first minimum corresponds to the suppressed diffusion scenario found in previous analyses of the HAWC data. In such scenario the extension of the $\gamma$-ray halo is linked to the diffusion radius $r_{d} \sim \sqrt{4 D t_{\text {loss }}}$ [18], which for the $e^{ \pm}$of energy $20-200 \mathrm{TeV}$ relevant for the HAWC data and for such low $D_{0}$ is of a few pc, and the $\gamma$-ray morphology is influenced by the $\exp \left(-r^{2} / r_{d}^{2}\right)$ that appears in the diffusive solution. This explains the strong dependence of the best -fit $D_{0}$ on the value of $B$, where $D_{0}$ varies by a factor of 10 when moving from $2 \mu \mathrm{G}$ to $4 \mu \mathrm{G}$. Indeed, a lower(larger) value of $B$ implies a smaller(larger) energy loss rate that has to be compensated by a smaller(larger) diffusion coefficient in order to reproduce the observed source extension. The second minimum corresponds to a scenario where the quasi-ballistic propagation dominates up to few tens of parsec from the source, and the transition between the two regimes shapes the surface brightness. An additional increase of $D_{0}$ changes only slightly the the $\chi^{2}$ since in the quasi-ballistic regime the radial profile is not much affected by the specific value of $D_{0}$. A similar trend of the $\chi^{2}$ is obtained also for Monogem but with much less prominent variations: we find a first minimum at about $2-3 \cdot 10^{25} \mathrm{~cm}^{2} / \mathrm{s}$ and a second minimum at $4-8 \cdot 10^{27} \mathrm{~cm}^{2} / \mathrm{s}$. Remarkably the best-fit value obtained for both sources in the ballistic-diffusive model is close to the values found by fitting CR data, and does not depend dramatically on $B$ and in the suppressed diffusion scenario. Moreover, the goodness of the fit for our model, with $\chi^{2} \sim 22$ for Geminga and $\sim 9$ for Monogem, indicates that this second minimum gives a satisfactory fit to the data.

In is important to highlight that such values of the $\chi^{2}$ are overestimated in the case of the ballistic-diffusive model. Indeed, looking at the data points for Geminga, for which we get the larger $\chi^{2}$, the obtained value is mainly dominated by the first data point at $\approx 0.32^{\circ}$, which corresponds to a distance from the source of 1.1-1.4 pc (for source distances of 190-250 pc, respectively). At such 

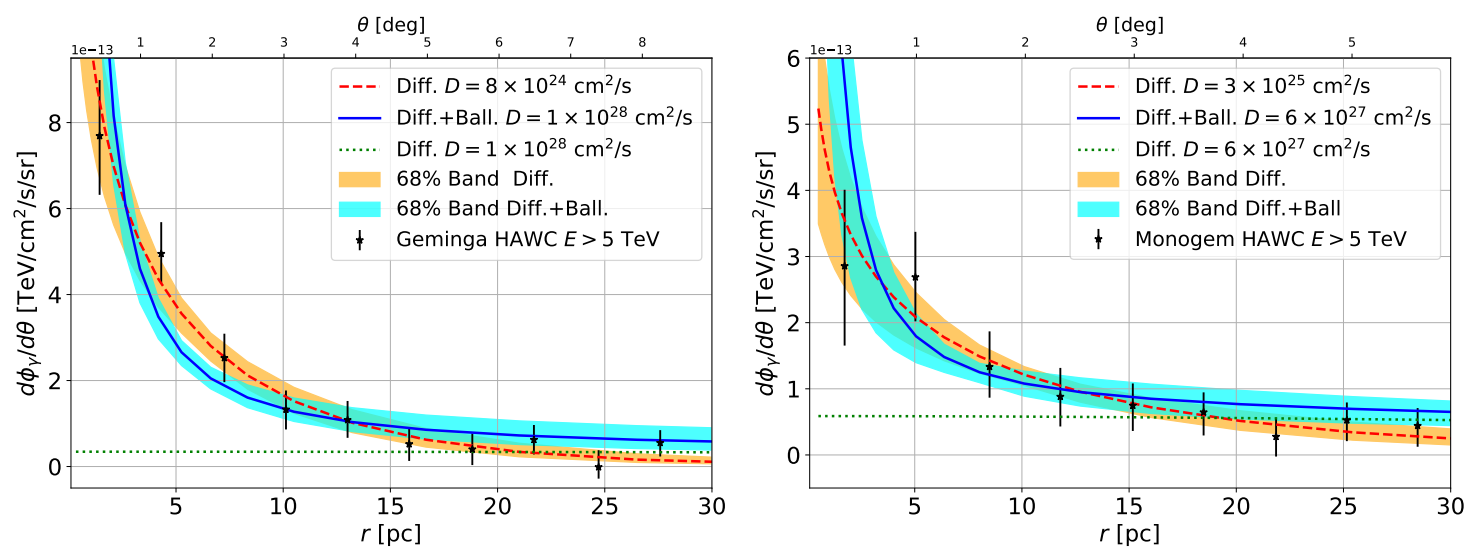

Figure 2: Fit to the HAWC data for Geminga (left panel) and Monogem (right panel) in the pure diffusive regime (red dotted line) and in the combined diffusive and ballistic model (blue solid line and cyan band). We adopt a distance for Geminga of $0.19 \mathrm{kpc}$ and for Monogem of $0.288 \mathrm{kpc}$.

small distances two factors have to be taken into account. First of all the size of the bow shock, which is likely of the order of a fraction of pc [20]. Our model is evidently not applicable inside the accelerator. Here we are assuming a bow-shock size of $0.3 \mathrm{pc}$, but an increase of such value would flatten our predictions at the first data point. Second, the convolution with the PSF of the experiment $\left(\approx 0.5^{\circ}[21]\right)$ is crucial in the quasi-ballistic regime, since it would flatten the rather sharp profile close to the source. In the pure ballistic model such effects do not change the shape substantially, since the profile is already quite flat close to the source. On the other hand, it is not possible to properly take into account the PSF without the analysis tools of the HAWC collaboration. Keeping these caveats in mind, the current precision of the data does not allow to discriminate statistically between the low and typical diffusion scenarios. However, since a typical diffusion coefficient can reproduce the data quite well, there is no theoretical motivation to invoke a suppression of diffusion coefficient that is difficult to explain.

The best-fit efficiency is much different in the low diffusion and ballistic-diffusion scenarios. Indeed in the first case $L_{e}$ is rather flat, while the second give $L_{e} \sim 1 / r$ (see Fig. 1). Consequently the required efficiency is smaller in the first case. For Geminga we get an efficiency of $0.3-3 \%$ in the low diffusion coefficient scenario and $55-65 \%$ for the quasi-ballistic one, depending on the value of $B$ and on the source distance. For the benchmark case of $B=3 \mu \mathrm{B}$ the efficiency for the diffusive and quasi-ballistic cases are 1.1\% [5] and 60\%, respectively. For Monogem the efficiency is about $1 \%$ and about $30 \%$, respectively. The spectral shape of the $e^{ \pm}$injected from PWNe, which is quite uncertain, can of course affect the efficiency. In particular changing the slopes below and above the break by \pm 0.2 and the break position from 200 to $1000 \mathrm{GeV}$ the efficiency for the quasi-ballistic case varies between 50-100\%. Such a high efficiency is in good agreement with the PWN paradigm, where a major fraction of the spin-down luminosity is channeled to multi-TeV $e^{ \pm}$ [22]. In particular, in the case of the Crab Nebula, $\eta$ is estimated to be close to $50 \%$. 


\section{Conclusions}

We demonstrate that at the $e^{ \pm}$energies relevant for the HAWC $\gamma$-ray data for Geminga and Monogem, and for typical values of the interstellar diffusion coefficient, the surface brightness, up to distances from the source of the order of $\lambda_{c}$ (which is few tens pc at the relevant energies), is dominated by particles that move in the quasi-ballistic regime. When the transition between ballistic and diffusive regimes is considered, it is possible to fit the HAWC data for Geminga and Monogem with typical values of the diffusion coefficient used to fit CR data, without the need of a suppressed diffusion, which is hard to justify theoretically. The current precision of the HAWC data does not allow to disentangle between a suppressed diffusion and a typical diffusion scenario. However, the possible future detection of the surface brightness of other sources, with different distances and ages compared to Geminga and Monogem, by HAWC and LHAASO [23] could allow to discriminate between the two. Anyway, our analysis indicated that, in general, $\gamma$-ray halos of continuous sources (not necessarily pulsar) should be analyzed with care, since a pure diffusion model may lead to wrong conclusions.

\section{References}

[1] HAWC collaboration, Extended gamma-ray sources around pulsars constrain the origin of the positron flux at Earth, Science 358 (2017) 911 [1711.06223].

[2] D. Hooper, I. Cholis, T. Linden and K. Fang, HAWC Observations Strongly Favor Pulsar Interpretations of the Cosmic-Ray Positron Excess, Phys. Rev. D 96 (2017) 103013 [1702.08436].

[3] X. Tang and T. Piran, Positron flux and $\gamma$-ray emission from Geminga pulsar and pulsar wind nebula, Mon. Not. Roy. Astron. Soc. 484 (2019) 3491 [1808.02445].

[4] K. Fang, X.-J. Bi, P.-F. Yin and Q. Yuan, Two-zone diffusion of electrons and positrons from Geminga explains the positron anomaly, Astrophys. J. 863 (2018) 30 [1803. 02640].

[5] M. Di Mauro, S. Manconi and F. Donato, Detection of a $\gamma$-ray halo around geminga with the fermi -lat data and implications for the positron flux, Phys. Rev. D 100 (2019) 123015 [1903.05647].

[6] M. Di Mauro, S. Manconi and F. Donato, Evidences of low-diffusion bubbles around galactic pulsars, Phys. Rev. D 101 (2020) 103035 [1908.03216].

[7] G. Giacinti, A.M.W. Mitchell, R. López-Coto, V. Joshi, R.D. Parsons and J.A. Hinton, Halo fraction in TeV-bright pulsar wind nebulae, Astron. Astrophys. 636 (2020) A113 [1907.12121].

[8] A.W. Strong, I.V. Moskalenko and V.S. Ptuskin, Cosmic-Ray Propagation and Interactions in the Galaxy, Annual Review of Nuclear and Particle Science 57 (2007) 285 [astro-ph/0701517]. 
[9] R. López-Coto and G. Giacinti, Constraining the properties of the magnetic turbulence in the Geminga region using HAWC $\gamma$-ray data, Mon. Not. Roy. Astron. Soc. 479 (2018) 4526 [1712.04373].

[10] C. Evoli, T. Linden and G. Morlino, Self-generated cosmic-ray confinement in TeV halos: Implications for TeV $\gamma$-ray emission and the positron excess, Phy. Rev. D 98 (2018) 063017 [1807.09263].

[11] R.-Y. Liu, H. Yan and H. Zhang, Understanding the Multiwavelength Observation of Geminga's Tev Halo: The Role of Anisotropic Diffusion of Particles, Phys. Rev. Lett. 123 (2019) 221103 [1904 . 11536].

[12] R. Aloisio and V.S. Berezinsky, Anti-GZK Effect in Ultra-High-Energy Cosmic Ray Diffusive Propagation, ApJ 625 (2005) 249 [astro-ph/0412578].

[13] A.Y. Prosekin, S.R. Kelner and F.A. Aharonian, Transition of propagation of relativistic particles from the ballistic to the diffusion regime, Phy. Rev. D 92 (2015) 083003 [1506.06594].

[14] M. Di Mauro, F. Donato and S. Manconi, On the interpretation of the latest ams-02 cosmic ray electron spectrum, .

[15] J.M. Yao, R.N. Manchester and N. Wang, A New Electron-density Model for Estimation of Pulsar and FRB Distances, ApJ 835 (2017) 29 [1610.09448].

[16] D.F. Torres, A. Cillis, J. Martín and E. de Oña Wilhelmi, Time-dependent modeling of TeV-detected, young pulsar wind nebulae, JHEAp 1-2 (2014) 31 [1402 . 5485].

[17] S. Gabici and F.A. Aharonian, Pointlike Gamma Ray Sources as Signatures of Distant Accelerators of Ultrahigh Energy Cosmic Rays, PRL 95 (2005) 251102 [astro-ph/0505462].

[18] S. Recchia, S. Gabici, F.A. Aharonian and J. Vink, Local fading accelerator and the origin of TeV cosmic ray electrons, Phy. Rev. D 99 (2019) 103022 [1811.07551].

[19] J.M. Cordes and T.J.W. Lazio, NE2001.I. A New Model for the Galactic Distribution of Free Electrons and its Fluctuations, arXiv e-prints (2002) astro [astro-ph/0207156].

[20] P.A. Caraveo, G.F. Bignami, A. DeLuca, S. Mereghetti, A. Pellizzoni, R. Mignani et al., Geminga's tails: A pulsar bow shock probing the interstellar medium, Science 301 (2003) 1345 [https://science. sciencemag.org/content/301/5638/1345 . full .pdf].

[21] A.U. Abeysekara et al., Observation of the Crab Nebula with the HAWC Gamma-Ray Observatory, Astrophys. J. 843 (2017) 39 [1701.01778].

[22] C.F. Kennel and F.V. Coroniti, Magnetohydrodynamic model of Crab nebula radiation., ApJ 283 (1984) 710.

[23] LHAASO collaboration, Performance of LHAASO-WCDA and Observation of Crab Nebula as a Standard Candle, arXiv e-prints (2021) arXiv:2101.03508 [2101.03508]. 